\section{Tratamiento inicial y único del edema macular asociado a oclusiones de rama venosa retiniana con fármacos antiangiogénicos. Estudio piloto comparativo}

\section{Initial and unique treatment of macular} edema due to branch retinal vein occlusion with antiangiogenic agents. A comparative pilot study

\section{Sr. Director:}

El edema macular (EM) constituye la causa principal de pérdida de visión asociada oclusión de rama venosa retiniana no isquémica (ORVR-NI) (1). Se han ensayado diversos tratamientos como la fotocoagulación en rejilla macular, la triamcinolona intravítrea, la vitrectomía asociada a pelado de la membrana limitante interna o disección de la adventicia arteriovenosa, con resultados variables y no exentos de complicaciones y/o efectos secundarios. El VEGF es fundamental en la patogenia del EM asociado a ORVR-NI (2), por lo que su inhibición mediante la inyección intravítrea de fármacos anti-VEGF constituye una potencial diana terapéutica (3), sin que haya sido establecido si un fármaco paninhibidor de todas las isoformas del VEGF-A podría ser superior a otro que inhibiera selectivamente la isoforma 165 de éste.

El objetivo del presente estudio ha sido valorar de manera comparativa la eficacia de dos fármacos antiangiogénicos (Pegaptanib y Ranibizumab) como tratamiento inicial y único del EM asociado a ORVR-NI.

Un total de 12 pacientes con EM secundario a ORVR-NI y grosor macular superior a 400 micras, fueron asignados de manera aleatoria consecutiva al grupo de tratamiento con Pegaptanib y al de Ranibizumab. El protocolo de tratamiento consistió en inyectar una primera dosis del correspondiente fármaco y retratar con una periodicidad de 6 semanas aquellos casos en que el grosor macular permaneciera por encima de las 300 micras. Los pacientes fueron reevaluados cada 6 semanas hasta completar un período de seguimiento de 30 semanas.

Con Pegaptanib $(n=6)$ la mejor agudeza visual corregida (MAVC) media mejoró desde logMAR $0,617 \pm 0,17$ hasta $0,367 \pm 0,10(\mathrm{p}=0,026)$; el grosor macular medio disminuyó desde 498,83 \pm 86,37 micras hasta $266,33 \pm 20,70(\mathrm{p}=0,028)$. A las 30 semanas de seguimiento fue necesaria una media de 3,3 inyecciones por paciente (fig. 1). Con Ranibizumab $(n=6)$ la MAVC media mejoró desde logMAR $0,717 \pm 0,19$ hasta $0,351 \pm 0,21(\mathrm{p}=0,026)$; el grosor macular medio disminuyó desde 545,67 \pm 87,66 micras hasta $260,67 \pm 31,76(p=0,028)$. A las 30 semanas de seguimiento fue necesaria una media de 2,3 inyecciones por paciente (fig. 2). No se detectaron diferencias estadísticamente significativas entre ambos grupos en cuanto a mejoría de MAVC $(\mathrm{p}=0,715)$, disminución del grosor macular $(\mathrm{p}=0,173)$ ni número de tratamiento $(\mathrm{p}=0,141)$. Todos los pacientes en los que se consiguió reducir por debajo de 300 micras todo el mapa de grosor macular (protocolo de análisis Retinal Map Thickness) permanecieron estables sin incremento en el EM a lo largo del seguimiento y no precisaron ulteriores retratamientos posteriores hasta las 30 semanas de seguimiento.

A la luz de nuestros resultados preliminares, podemos sugerir que el tratamiento del EM asociado a ORVR-NI realizado de manera inicial y único con fármacos antiangiogénicos resultan de similar eficacia el Pegaptanib (Macugen ${ }^{\circledR}$ ) como el Ranibizumab (Lucentis ${ }^{\circledR}$ ). Estudios multicéntricos rando-

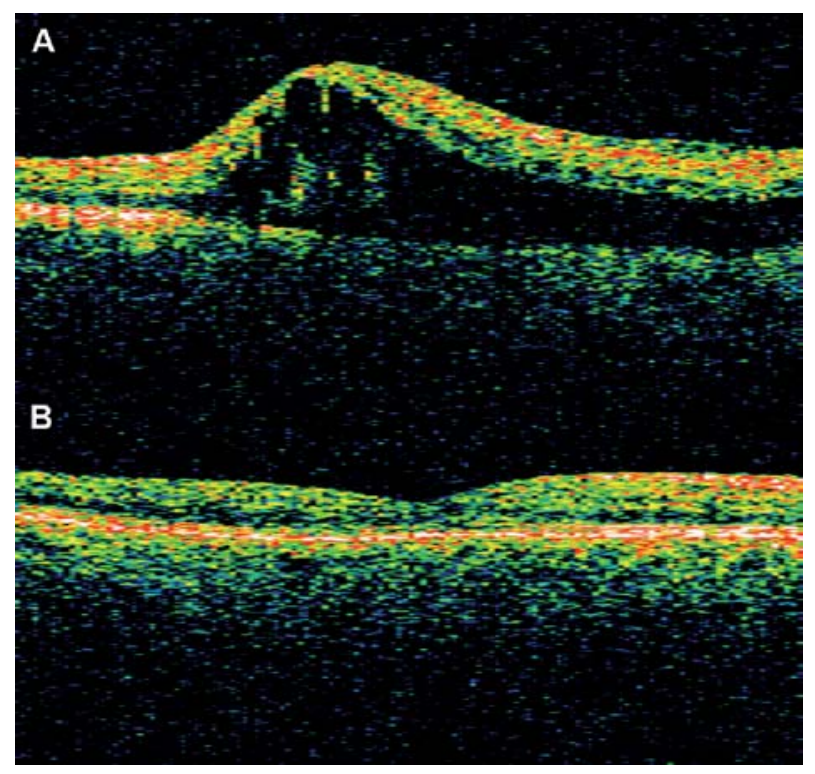

Fig. 1: EM secundario a ORVR-NI (A) tratado con 3 inyecciones de Pegaptanib (Macugen $\left.{ }^{\circledR}\right)$ (B). Mejoría desde 712 micras iniciales a 275 micras. La visión mejoró desde 0,7 logMAR (equivalente decimal escala Snellen: 0,20) a 0,5 logMAR (equivalente decimal escala Snellen: 0,32). 


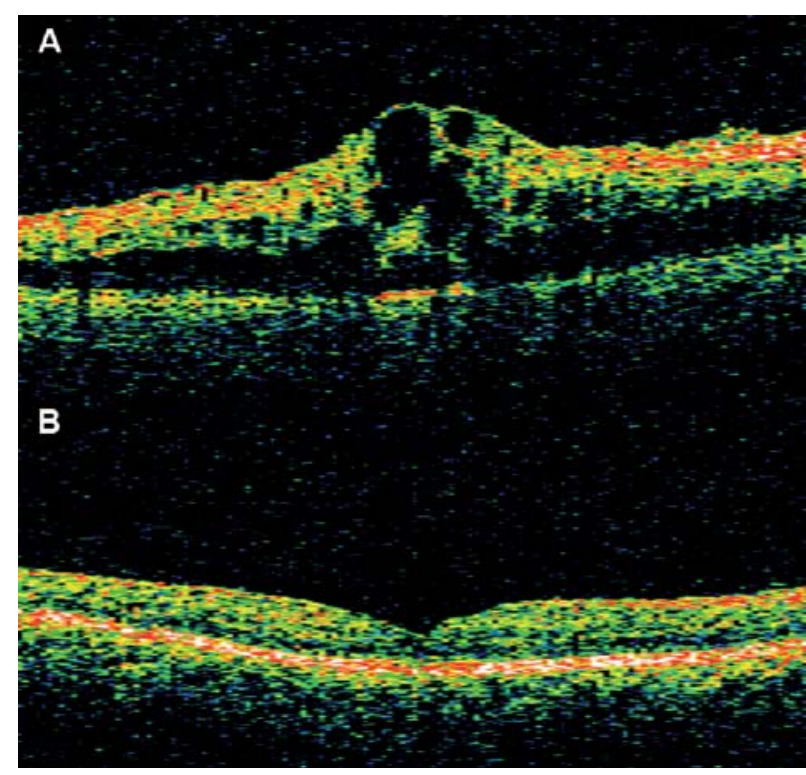

Fig. 2: EM asociado a ORVR-NI (A) tratado con 2 inyecciones de Ranibizumab (Lucentis $\left.{ }^{\circledR}\right)(B)$. Mejoría desde 881 micras a 283 micras. La visión pasó de 0,80 logMAR (equivalente decimal escala Snellen: 0,02) a 0,2 logMAR (equivalente decimal escala Snellen: 0,63). mizados con mayor número de casos y tiempo de seguimiento deberán corroborar las conclusiones de este estudio piloto.

Gallego-Pinazo $\mathrm{R}^{1}$, Francés $\mathrm{E}^{1}$, Gómez $\mathrm{MJ}^{1}$, López $\mathrm{R}^{1}$, Díaz-Llopis $\mathrm{M}^{1,2}$ 1 Servicio de Oftalmología. Hospital Universitario La Fe. Valencia.

2 Universidad de Valencia. Facultad de Medicina E-mail: robertogallego@comv.es

\section{BIBLIOGRAFÍA}

1. Klein R, Klein BE, Moss SE, Meuer SM. The epidemiology of retinal vein occlusion: the Beaver Dam Eye Study. Trans Am Ophthalmol Soc 2000; 98: 133-141.

2. Noma H, Funatsu H, Yamakasi M, Tsukamoto H, Mimura $T$, Sone T, et al. Pathogenesis of macular edema with branch retinal vein occlusion and intraocular levels of vascular endothelial growth factor and interleukin-6. Am J Ophthalmol 2005; 140: 256-261.

3. Jaissle GB, Leiritz M, Gelisken F, Ziemssen F, BartzSchmidt KU, Szuman P. One-year results after intravitreal bevacizumab therapy for macular edema secondary to branch retinal vein occlusion. Graefes Arch Clin Exp Ophthalmol 2009; 247: 27-33. 